\title{
Nonicosahedral pathways for capsid expansion
}

\author{
Paolo Cermelli, ${ }^{1, *}$ Giuliana Indelicato, ${ }^{2, \dagger}$ and Reidun Twarock ${ }^{2, \ddagger}$ \\ ${ }^{1}$ Dipartimento di Matematica, Università di Torino, Torino, Italy \\ ${ }^{2}$ York Centre for Complex Systems Analysis, Department of Mathematics, University of York, York, United Kingdom
}

(Received 24 January 2013; revised manuscript received 26 June 2013; published 16 September 2013)

\begin{abstract}
For a significant number of viruses a structural transition of the protein container that encapsulates the viral genome forms an important part of the life cycle and is a prerequisite for the particle becoming infectious. Despite many recent efforts the mechanism of this process is still not fully understood, and a complete characterization of the expansion pathways is still lacking. We present here a coarse-grained model that captures the essential features of the expansion process and allows us to investigate the conditions under which a viral capsid becomes unstable. Based on this model we demonstrate that the structural transitions in icosahedral viral capsids are likely to occur through a low-symmetry cascade of local expansion events spreading in a wavelike manner over the capsid surface.
\end{abstract}

DOI: 10.1103/PhysRevE.88.032710

PACS number(s): 87.10.Ca, 87.15.bk

\section{INTRODUCTION}

Viruses are important examples of symmetry in biology. This is due to the principle of genetic economy [1,2], which suggests that protein containers built from multiple copies of identical building blocks guided by symmetry principles can be coded for by relatively short genomic sequences, hence making genome packaging efficient. Symmetry has therefore dominated the field of virus structure for decades and is the bedrock of imaging techniques that provide insights into capsid structures with remarkable precision. However, it has recently become increasingly clear that asymmetric components play crucial roles for virus function, e.g., for the mechanisms of genome release. Therefore, any models trying to understand how viruses form and infect their hosts must take such asymmetric components into account. An example of such an asymmetric feature is the single copy of maturation protein in bacteriophage MS2, which may replace one of the capsomeres (in this virus protein dimers) and play an essential role in genome ejection at that site.

On the other hand, even when the capsid is fully symmetric, conformational changes are often triggered by variations of its chemical environment that, in turn, modify the interaction energies between the proteins. The effect of such changes is most likely nonuniform, in the sense that they induce fluctuations in the interactions that may be stronger at specific random sites of the capsid.

We present here a coarse-grained model that allows us to study the effect of nonuniform intercapsomere bonds on the transition path during the expansion of the capsid, either due to, e.g., the insertion of asymmetric features in the capsid or to random local fluctuations of the bond energy induced by variations of the environment.

Structural transitions occur in many viruses, for example, in a large number of plant viruses [3-5]. Since these have devastating effects on agriculture, a better understanding

\footnotetext{
*paolo.cermelli@unito.it

†'giuliana.indelicato@unito.it

†reidun.twarock@york.ac.uk
}

of these transition mechanisms and the potential roles of asymmetric components in this process may open up new insights in how to block such transitions, and could lead to the design of antiviral strategies. Structural transitions of viral capsids have been studied extensively both in the theoretical and the experimental literature [6]. A prominent example is bacteriophage HongKong97 (HK97), which undergoes a maturation process that involves an expansion of the capsid [7-9]. Different experimental techniques have been used to study such capsid expansions, including cryoelectron microscopy [10] and atomic force microscopy [11], and elastic network models have been used to study these transitions $[12,13]$. While bacteriophage HK97 is perhaps the most widely studied system, viruses with other capsid geometries, such as cowpea chlorotic mottle virus (CCMV), have also been studied [14,15].

An important common feature of these transitions is the importance of subunit rotations [16]. We therefore construct here a model system to study the mechanism by which such subunit rotations may occur along the transition path. A model system often used to study mechanisms underlying vital stages of the viral life cycle is the dodecahedron, a shape formed from 12 regular pentagons. It provides a simple geometric representation of an icosahedral capsid, yet captures all its essential features. For example, it has been used to model the principles of capsid self-assembly from 12 pentagonal protein clusters $[17,18]$. Even though the dodecahedron provides a geometric idealization, there are viruses for which the structural transition in the protein container occurs via a rigid body movement of pentagonal protein clusters, e.g., equine rhinitis A virus (ERAV [19]), and our model is therefore directly applicable to them (Fig. 1). However, the purpose of this paper is to investigate the physical determinants of such transitions in general terms and to show that in the case of nonuniform intercapsomere bonds the capsid opening occurs via a cascade of localized detachment events propagating along the capsid surface in a wavelike motion.

We show here that this wavelike expansion is a consequence of a competition between the attractive forces between protein interfaces and a repulsive force exerted by the packaged genome. Such an opening scenario is plausible given that, 


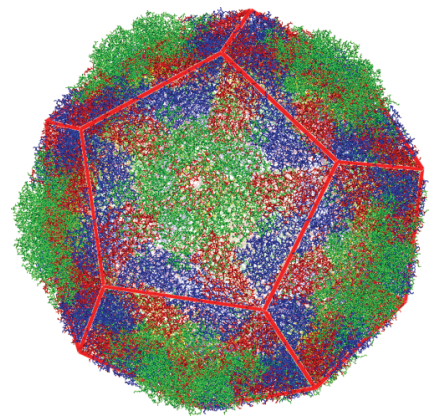

FIG. 1. (Color online) Equine rhinitis A virus (ERAV) follows the geometry of our dodecahedral model system.

as we show here, the energy required to initiate opening at localized interfaces is far less than that required for the capsid to open instantaneously as a whole. Local variations in the bond strengths could easily be triggered either by asymmetric components impacting on specific subsets of intercapsomere bonds or by local fluctuations in solution conditions favoring certain bonds over others. We also show that even if a transition path breaks icosahedral symmetry, the final state of the expansion must be icosahedral again. Our analysis hence predicts icosahedral configurations (modulo localized asymmetric components) before and after transition. The lower intermediate symmetry during transitions depends on the number and locations of the weakened intercapsomere bonds.

Even though we consider explicitly the rigid motion of pentagonal protein building blocks in the context of an idealized dodecahedral capsid, the conclusions transfer to other scenarios of rigid body motion and are hence valid for larger classes of viruses. Examples of such rigid body motion are well known and can also involve rigid motion of protein domains $[5,16]$. In viruses where the units of rigid motion are not known a priori, domain decomposition techniques based on a normal mode analysis can help to determine them [20]. Moreover, an analysis of the atomic positions of the capsid proteins (pdb files available, e.g., from VIPER [21]) makes it possible to characterize the rigid domains of a virus of interest in terms of their center of mass and contact map. It is possible to add a further layer of complexity to our models by using the available libraries of empirical potentials to approximate the interactions between capsid proteins, which would hence customize this analysis to specific viruses of interest. However, it is the purpose of this paper to demonstrate in general terms in a coarse-grained setting that a wavelike cascade of localized low-symmetry opening events is the most likely scenario for viral capsid transitions and to provide a framework that allows the impact of a nonhomogeneous distribution of intercapsomere bonds, e.g., caused by asymmetric components or solution conditions, on structural transitions in viral capsids to be studied.

Since it explicitly takes into account the interactions among the proteins, our work may complement either existing phenomenological approaches based on continuum elasticshell models [22-24] or coarse-grained models similar to ours but where icosahedral symmetry is imposed on the transition intermediates [25].
The paper is organized as follows. We start with a section introducing the model system and the variables that describe it. This section also introduces the energy function and model assumptions. In the subsequent section, we analyze the triggers of the capsid expansion event, discussing the symmetric case in which all bond strengths are assumed to be equal and the asymmetric case in which one bond strength differs from the others.

We then show that in these cases capsid expansion is likely to occur as a wavelike cascade of local expansion events, involving rotation and radial expansion of individual capsomeres. This section is followed by a discussion of the nature of the end point of the expansion. We conclude by discussing the implications of the results for wider classes of viruses.

\section{THE MODEL SYSTEM}

We consider a dodecahedral model system [see Fig. 2(a)] in which the pentagonal faces $F_{i}, i=1, \ldots, 12$, act as rigid units during the transition. We refer to $F_{i}$ as the pentamers, even though, in actual dodecahedral capsids, each subunit can be composed of multiples of five proteins.

Information on adjacency of the pentamers is captured by the adjacency set $A=\{(i, j)$ :pentamer $i$ is adjacent to pentamer $j\}, i<j\}$ and the adjacency matrix

$$
A_{i j}= \begin{cases}1 & \text { when }(i, j) \in A, \\ 0 & \text { otherwise. }\end{cases}
$$

Moreover, $N(i)$ indicates the set of faces adjacent to pentamer $i$.

The motion of pentamer $F_{i}$ during the transition is characterized by its radial displacement $\lambda_{i}$ along, and rotation $\theta_{i}$ about the unit vector $\boldsymbol{n}_{i}$ orthogonal to its plane and passing through the center of the dodecahedron [Fig. 2(b)], i.e., by $F_{i} \mapsto R_{i}\left(\vartheta_{i}\right) F_{i}+\lambda_{i} \boldsymbol{n}_{i}$, where

$R_{i}\left(\vartheta_{i}\right) \boldsymbol{v}=\left(\boldsymbol{v} \cdot \boldsymbol{n}_{i}\right) \boldsymbol{n}_{i}+\cos \vartheta_{i}\left[\boldsymbol{v}-\left(\boldsymbol{v} \cdot \boldsymbol{n}_{i}\right) \boldsymbol{n}_{i}\right]+\sin \vartheta_{i}\left(\boldsymbol{n}_{i} \times \boldsymbol{v}\right)$,

for any vector $\boldsymbol{v}$. The system is hence entirely characterized by the 24 degrees of freedom $(\boldsymbol{\lambda}, \boldsymbol{\vartheta})=\left(\lambda_{i}, \vartheta_{i}\right), i=1, \ldots, 12$, with $\lambda_{i} \in[0,+\infty)$ and $\vartheta_{i} \in(-\pi, \pi)$ for $i=1, \ldots, 12$. We do not impose here any constraint preventing the interpenetration of the pentamers other than the requirement that $\lambda_{i} \geqslant 0$. Such constraints can be introduced by restricting the domain of the relevant variables but would not change the analysis below.

In order to keep the integrity of the capsid after expansion, we assume that the pentamers are linked by the C- or N-

(a)

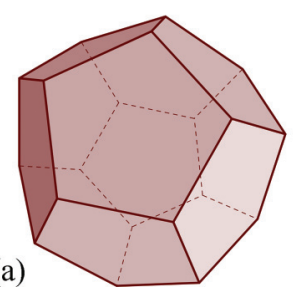

(b)

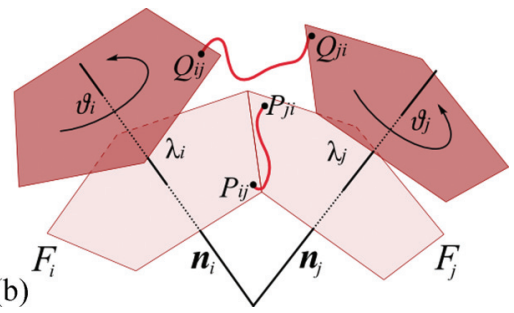

FIG. 2. (Color online) (a) The dodecahedral model system. The translational $(\lambda)$ and rotational $(\vartheta)$ degrees of freedom of each pentamer, and the links between them, are illustrated in (b). 
terminal arms of the proteins, which we model here as elastic but almost inextensible threads. For simplicity we assume that there is only one chain linking each adjacent pair of pentamers and that the anchor points $\boldsymbol{P}_{i j} \in F_{i}$ and $\boldsymbol{P}_{j i} \in F_{j}$ of this chain in the closed reference configuration are symmetric with respect to the common edge of the pentamers; see Fig. 2(b). As the pentamers move, so do the anchor points: denoting by $\boldsymbol{Q}_{i j}\left(\lambda_{i}, \vartheta_{i}\right)$, respectively $\boldsymbol{Q}_{j i}\left(\lambda_{j}, \vartheta_{j}\right)$, the positions of the anchor points $\boldsymbol{P}_{i j}$ and $\boldsymbol{P}_{j i}$ after displacement of the pentamers by $\left(\lambda_{i}, \vartheta_{i}\right)$ (pentamer $\left.i\right)$ and $\left(\lambda_{j}, \vartheta_{j}\right)$ (pentamer $\left.j\right)$, the distance between the displaced anchor points is denoted by

$$
\ell_{i j}:=\ell\left(\lambda_{i}, \lambda_{j}, \vartheta_{i}, \vartheta_{j}\right):=\left|\boldsymbol{Q}_{i j}-\boldsymbol{Q}_{j i}\right| .
$$

Hence, the constraint that the pentamers $F_{i}$ and $F_{j}$ are linked by a chain between the displaced anchor points $\boldsymbol{Q}_{i j}$ and $\boldsymbol{Q}_{j i}$ takes the form

$$
0 \leqslant \ell\left(\lambda_{i}, \lambda_{j}, \vartheta_{i}, \vartheta_{j}\right) \leqslant \ell_{0} .
$$

Here $\ell_{0}>\left|\boldsymbol{P}_{i j}-\boldsymbol{P}_{j i}\right|=\ell(0,0,0,0)$ is the rest length of the arm connecting two pentamers, which we assume to be strictly larger than the initial distance between the points in the closed configuration. This is to account for the fact that $\mathrm{C}$ or N-terminal arm connections are not tight and allow for some give in the distance between the pentamers. To avoid characterizing the constraint analytically, we relax (1) by introducing an energy term penalizing its violation.

\section{A. The energy function orchestrating the transition}

We assume that the total energy of the capsid has the form

$$
E(\boldsymbol{\lambda}, \boldsymbol{\vartheta})=W_{\text {blk }}(\boldsymbol{\lambda})+W_{\text {int }}(\boldsymbol{\lambda}, \boldsymbol{\vartheta})+W_{\text {constr }}(\boldsymbol{\lambda}, \boldsymbol{\vartheta})
$$

and is given in terms of the following components.

(i) RNA expansion (expansive pressure from the RNA),

$$
W_{\mathrm{blk}}=\sum_{i=1}^{12} G\left(\lambda_{i}\right)
$$

with $G:[0,+\infty) \rightarrow \mathbb{R}$ a smooth decreasing function such that $G(x)>0, G(x) \rightarrow 0$ as $x \rightarrow+\infty, G^{\prime}(x)<0$, and $G^{\prime}(x)$ is monotone increasing. $W_{\mathrm{blk}}$ accounts for a radial repulsion force due to RNA confinement (Fig. 3).

(ii) The cohesive interfacial energy,

$$
W_{\mathrm{int}}=\frac{1}{2} \sum_{(i, j) \in A} \alpha_{i j} F\left(d\left(\lambda_{i}, \lambda_{j}, \vartheta_{i}, \vartheta_{j}\right)\right), \quad \alpha_{i j} \in[0,1],
$$

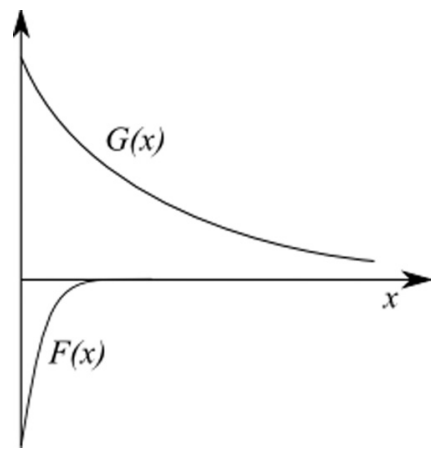

FIG. 3. Qualitative plots of the RNA-capsid and intercapsomere interaction energy functions. with $F:[0,+\infty) \rightarrow \mathbb{R}$ a smooth increasing function such that $F(x)<0, F(x) \rightarrow 0$ as $x \rightarrow+\infty, F^{\prime}(x)>0, F^{\prime}(x)$ is monotone decreasing. We assume that $F^{\prime}(x)$ goes to zero much faster than $G^{\prime}$ to account for the fact that interfacial energies ebb off faster with distance than the radial outwards push of the RNA (Fig. 3). The weight $\alpha_{i j}$ is the strength of the bond $(i, j) \in A$.

The function $d\left(\lambda_{i}, \lambda_{j}, \vartheta_{i}, \vartheta_{j}\right)$ in (3) is a measure of the distance between adjacent pentameric building blocks. The exact expression is not required for our line of arguments, but we assume that $d$ is symmetric and positive definite, i.e., $d\left(\lambda, \lambda^{\prime}, \vartheta, \vartheta^{\prime}\right)=d\left(\lambda^{\prime}, \lambda, \vartheta^{\prime}, \vartheta\right) \geqslant 0$, with $d\left(\lambda, \lambda^{\prime}, \vartheta, \vartheta^{\prime}\right)=0$ if and only if $\lambda=\lambda^{\prime}=\vartheta=\vartheta^{\prime}=0$. Moreover, we assume that

$$
\begin{aligned}
\Lambda:= & \left.\frac{\partial d}{\partial \lambda}\left(\lambda, \lambda^{\prime}, \vartheta, \vartheta^{\prime}\right)\right|_{(0,0,0,0)}>0 \text { and } \\
& \left.\frac{\partial d}{\partial \vartheta}\left(\lambda, \lambda^{\prime}, \vartheta, \vartheta^{\prime}\right)\right|_{\left(\lambda, \lambda^{\prime}, 0,0\right)}=0
\end{aligned}
$$

for $\lambda, \lambda^{\prime} \geqslant 0$. We also assume that the distance has a linear growth with $\lambda$, i.e., that there exist positive constants $c_{1}$ and $c_{2}$ for which

$$
c_{1}<\frac{\partial d}{\partial \lambda}\left(\lambda, \lambda^{\prime}, \vartheta, \vartheta^{\prime}\right)<c_{2},
$$

for all $\left(\lambda, \lambda^{\prime}, \vartheta, \vartheta^{\prime}\right)$ with $\lambda, \lambda^{\prime} \geqslant 0$. Notice that $(4)_{1}$ is implied by the above inequality.

(iii) The constraint energy,

$$
W_{\text {constr }}=C \sum_{(i, j) \in A}\left\{\begin{array}{lll}
\left(\ell_{i j}^{2}-\ell_{0}^{2}\right)^{n} & \text { if } \quad \ell_{i j}^{2}>\ell_{0}^{2}, \\
0 & \text { if } \quad \ell_{i j}^{2} \leqslant \ell_{0}^{2},
\end{array}\right.
$$

with $C$ a positive constant and $n \geqslant 2$, captures the fact that the motion of the pentamers is constrained by the $\mathrm{C}$ - or $\mathrm{N}$-terminal arm linking them.

We note that all conclusions of the paper hold true as long as we assume that there is some force that prevents pentamers from completely detaching from the capsid. The exact form of this term is hence irrelevant and could be replaced by any other form with this property.

The model is formulated in terms of an expansive force stemming from a radial repulsive force due to RNA confinement that is balanced by a cohesive force between capsomeres. We note that this is not appropriate for all types of viruses. For instance, in CCMV the driving force for capsid expansion is Coulomb repulsion between the capsid proteins, which is turned on by the removal of calcium ions. However, for other viruses, such as ERAV, the expansion is triggered by decrease of the $p \mathrm{H}$, rather than the removal of metal ions, and closed empty particles at low $p \mathrm{H}$ are experimentally observed [19,26], suggesting that, in this case, there is no repulsion between the capsomeres and our assumption about cohesive force is reasonable.

Further, RNA is negatively charged and binds to positively charged capsid proteins, which helps the assembly in many RNA viruses, since proteins attach at RNA stem-loops during the assembly. This attractive force between the RNA and the capsomeres is not in contrast with the radial repulsive force due to confinement which we assume to promote capsid expansion. 


\section{B. Model assumptions}

We study the dynamics of the system based on the following hypotheses.

(i) Hypothesis I. When the expansive force on a pentamer overcomes the cohesive force between it and its neighbors, the pentamer detaches, but otherwise remains in its initial position. In other words, the closed capsid is stable as long as the closed configuration is in a minimum of the total energy.

(ii) Hypothesis II. When a pentamer moves, it does so according to a dissipative mechanism; i.e., it tends to decrease the overall energy.

Since the closed configuration of the capsid corresponds to $\lambda=\mathbf{0}, \boldsymbol{\vartheta}=\mathbf{0}$, which is a boundary point of the domain of definition of the energy, the minimum condition should be restated in terms of the subdifferential of the energy. In our case, it turns out to be simpler to identify the partial derivatives $-\partial E / \partial \lambda_{i}$ and $-\partial E / \partial \vartheta_{i}$ with the generalized forces acting on the pentamers and, consistent with Hypothesis II, assume that the motion of each pentamer is governed by a gradient system. We hence obtain the following dynamical system:

$$
\dot{\lambda}_{i}= \begin{cases}0 & \text { if } \lambda_{i}=0 \text { and }-\frac{\partial E}{\partial \lambda_{i}}(\boldsymbol{\lambda}, \boldsymbol{\vartheta}) \leqslant 0, \\ -\frac{\partial E}{\partial \lambda_{i}}(\boldsymbol{\lambda}, \boldsymbol{\vartheta}) & \text { if } \lambda_{i}>0, \\ & \text { or } \lambda_{i}=0 \text { and }-\frac{\partial E}{\partial \lambda_{i}}(\boldsymbol{\lambda}, \boldsymbol{\vartheta})>0,\end{cases}
$$

and

$$
\dot{\vartheta}_{i}=-\frac{\partial E}{\partial \vartheta_{i}}(\lambda, \vartheta)
$$

for $i=1, \ldots, 12$.

The rationale behind $(6)_{1}$ is as follows: identifying $-\frac{\partial E}{\partial \lambda_{i}}(\boldsymbol{\lambda}, \boldsymbol{\vartheta})$ at $\lambda_{i}=0$ with the force opposing or favoring the expansion of the $i$ th pentamer in its initial configuration, a negative value implies that this force acts by keeping the capsid in the closed form, given the constraint $\lambda_{i} \geqslant 0$, i.e., that the capsid cannot contract further from the closed state. The capsid opens when the force is positive, i.e., when the expansive contribution overcomes the cohesive term.

\section{THE INITIATION OF THE EXPANSION}

We first explore the energetic cost of initiating capsid expansion, either at all interfaces simultaneously or at selected interfaces. As the constraint energy and its derivatives vanish in the closed form of the capsid, the stability conditions $(6)_{1}$, i.e.,

$$
-\frac{\partial E}{\partial \lambda_{i}}(\mathbf{0}, \mathbf{0}) \leqslant 0
$$

reduce to

$$
0 \leqslant G^{\prime}(0)+\Lambda F^{\prime}(0) \sum_{j \in N(i)} \alpha_{i j}, \quad i=1, \ldots, 12,
$$

where $\alpha_{i j}$ denote the interpentamer bond strengths. The above condition is equivalent to

$$
\begin{aligned}
\sum_{j \in N(i)} \alpha_{i j} & \geqslant 5 \alpha_{c} \quad \text { with } \quad \alpha_{c}:=-\frac{G^{\prime}(0)}{\Lambda F^{\prime}(0)}, \\
i & =1, \ldots, 12
\end{aligned}
$$

where $G^{\prime}(0)<0$. When one of the above inequalities is not satisfied for some pentamer $i$, the closed capsid is unstable. The stability condition (8) can be interpreted in terms of a balance of forces: we can identify the term $F_{\text {blk }}=-\frac{\partial W_{\text {blk }}}{\partial \lambda_{i}}(\mathbf{0 , 0})=$ $-G^{\prime}(0)$ with the expansive force acting on each pentamer and the term $F_{\text {int,i }}=-\frac{\partial W_{\text {int }}}{\partial \lambda_{i}}(\mathbf{0 , 0})=-\Lambda F^{\prime}(0) \sum_{j \in N(i)} \alpha_{i j}$ with the cohesive force keeping the capsid together. Hence, rewriting (8) as

$$
\left|F_{\text {blk }}\right| \leqslant\left|F_{\text {int,i }}\right|
$$

we can reformulate the stability condition as saying that the pentamer $i$ does not detach when the cohesive force is larger than the expansive force acting on it.

The stability condition depends on the bond strengths $\alpha_{i j}$, and we discuss two special cases below.

Case 1: all bond strengths are equal. In this case, we have

$$
\alpha_{i j}=\alpha \quad \text { for } \quad(i, j) \in A, \quad \alpha_{i j}=0 \quad \text { otherwise },
$$

and the stability condition reduces to $\alpha \geqslant \alpha_{c}$.

When, e.g., due to a variation in the chemical environment, the bond strengths decrease uniformly and reach this threshold, the capsid undergoes expansion. The energy required to overcome the threshold starting from a given closed configuration, in which all bond strengths are equal to a given value $\alpha_{0}>\alpha_{c}$, is

$$
\begin{aligned}
E_{\alpha_{c}}(\mathbf{0}, \mathbf{0})-E_{\alpha_{0}}(\mathbf{0 , 0})= & {\left[12 G(0)+30 F(0) \alpha_{c}\right] } \\
& -\left[12 G(0)+30 F(0) \alpha_{0}\right] \\
= & -30 F(0)\left(\alpha_{0}-\alpha_{c}\right),
\end{aligned}
$$

which is positive since $F(0)<0$.

Case 2: one bond strength is lower than the others. Many viruses have asymmetric features in their capsids that play a role in disassembly and genome ejection. An example is bacteriophage MS2, with a capsid in which one of the protein dimers is substituted by a single copy of maturation protein [27]. The presence of such asymmetric features motivates the analysis of an asymmetric energy formulation, in which a small number of the inter-subunit bonds differs from the others. This case may also occur due to fluctuations of the bond strengths induced by changes of the environment.

We consider here explicitly the case in which one bond is weaker than the others. The overall conclusions also hold for other asymmetric scenarios as we discuss in the conclusions section. In this case we have, for instance,

$\alpha_{12}=\alpha_{21}=\alpha-\epsilon, \quad \epsilon>0, \quad$ and $\quad \alpha_{i j}=\alpha \quad$ otherwise,

for $(i, j) \in A$ and assume that $\alpha=\alpha_{0}>\alpha_{c}$. Instability arises if one of the inequalities (9) is not satisfied. Since $\alpha_{0}>\alpha_{c}$, this can only occur for $i=1,2$, i.e., when

$$
0>G^{\prime}(0)+\Lambda F^{\prime}(0)\left(5 \alpha_{0}-\epsilon\right) \Leftrightarrow \epsilon>\epsilon_{c}=5\left(\alpha_{0}-\alpha_{c}\right) .
$$

The above inequality is meaningful when $\alpha_{12}=\alpha_{0}-\epsilon>0$, which means that $\alpha_{c}<\alpha_{0}<\frac{5}{4} \alpha_{c}$, i.e., $\alpha_{0}$ is sufficiently close to $\alpha_{c}$. The energetic cost of decreasing the bond strength $\alpha_{12}$ 


$$
\begin{aligned}
& \text { from } \alpha_{0} \text { to } \alpha_{0}-\epsilon_{c} \text { is } \\
& \qquad \begin{aligned}
E_{\alpha_{0}-\epsilon_{c}}(\mathbf{0 , 0})-E_{\alpha_{0}}(\mathbf{0 , 0})= & {\left[12 G(0)+F(0)\left(30 \alpha_{0}-\epsilon_{c}\right)\right] } \\
& -\left[12 G(0)+30 F(0) \alpha_{0}\right] \\
= & -F(0) \epsilon_{c}=-5 F(0)\left(\alpha_{0}-\alpha_{c}\right) .
\end{aligned}
\end{aligned}
$$

Hence, the energetic cost of decreasing a single bond until destabilization is lower than the cost of decreasing all bonds until destabilization:

$$
E_{\alpha_{0}-\epsilon_{c}}(\mathbf{0 , 0})-E_{\alpha_{0}}(\mathbf{0 , 0})<E_{\alpha_{c}}(\mathbf{0 , 0})-E_{\alpha_{0}}(\mathbf{0 , 0}) .
$$

Therefore, from an energetic point of view, Case 2 is the more likely scenario.

\section{CAPSID TRANSITION AS A CASCADE OF LOCAL EXPANSION EVENTS}

We next determine explicitly a possible scenario for capsid expansion. We focus in this section on the initial stages of the expansion, where contributions from $W_{\text {constr }}$ can be neglected, because the maximum allowable length of the links between pentamers is much larger than their distance in the initial stages. In this case, by $(4)_{2}$ and (7), we may take $\vartheta_{i}(t) \equiv 0$ for $t<T$, where $T$ is the maximal time at which no link is stretched, i.e., $T=\sup \left\{t>0: \ell\left(\lambda_{i}(t), \lambda_{j}(t), 0,0\right)<\right.$ $\ell_{0}$ for all $\left.(i, j) \in A\right\}$.

In that case, we can limit considerations to the following reduced system (where the dependence on $\vartheta_{i}$ is omitted):

$$
\dot{\lambda}_{i}= \begin{cases}-\frac{\partial \tilde{E}}{\partial \lambda_{i}}(\lambda) & \text { if } \lambda_{i}>0, \\ \max \left(0,-\frac{\partial \tilde{E}}{\partial \lambda_{i}}(\boldsymbol{\lambda})\right) & \text { if } \lambda_{i}=0,\end{cases}
$$

with

$$
\tilde{E}=\tilde{E}(\boldsymbol{\lambda})=W_{\text {blk }}(\boldsymbol{\lambda})+W_{\text {int }}(\boldsymbol{\lambda}, \mathbf{0}) .
$$

Letting $H\left(\lambda, \lambda^{\prime}\right)=F^{\prime}\left(d\left(\lambda, \lambda^{\prime}, 0,0\right)\right) \frac{\partial d}{\partial \lambda}\left(\lambda, \lambda^{\prime}, 0,0\right)>0$, we can write

$$
\frac{\partial \tilde{E}}{\partial \lambda_{i}}=G^{\prime}\left(\lambda_{i}\right) \sum_{j \in N(i)} \alpha_{i j} H\left(\lambda_{i}, \lambda_{j}\right) .
$$

We consider explicitly the case in which one of the bond strengths is smaller than the others, i.e., the scenario described by (10) in Case 2 above. Assume that $\alpha_{12}=\alpha-\epsilon, \alpha_{i j}=\alpha$ otherwise, and that the conditions (10) for detachment are satisfied. Since the distribution of bond strengths $\alpha_{i j}$ is trivially twofold symmetric about the axis going through the center of the edge $F_{1} \cap F_{2}$ and the center of the particle, the 12 variables $\lambda_{i}$ describing the individual expansions along axes perpendicular to the pentamers reduce to five independent variables, and we introduce the following notation:

$$
\begin{aligned}
\mu(t) & :=\lambda_{1}(t)=\lambda_{2}(t), \\
\nu(t) & :=\lambda_{6}(t)=\lambda_{10}(t), \\
\sigma(t) & :=\lambda_{3}(t)=\lambda_{5}(t)=\lambda_{9}(t)=\lambda_{11}(t), \\
\tau(t) & :=\lambda_{4}(t)=\lambda_{12}(t), \\
\xi(t) & :=\lambda_{7}(t)=\lambda_{8}(t) .
\end{aligned}
$$

The dynamics of (11) with initial conditions $\lambda\left(t_{0}\right)=\mathbf{0}$ is as follows. Since, by $(10),-\frac{\partial \tilde{E}}{\partial \lambda_{1}}(\mathbf{0})=-\frac{\partial \tilde{E}}{\partial \lambda_{2}}(\mathbf{0})>0$ at $t=$ $t_{0}$, pentamers 1 and 2 detach from the capsid and then expand radially, but none of the other pentamers are mobile yet, since their stability conditions $-\frac{\partial \tilde{E}}{\partial \lambda_{j}}(\mathbf{0})<0, j \neq 1,2$, are still satisfied. However, as a consequence of this local radial expansion, the bonds between pentamers 1 and 2 and the adjacent pentamers are weakened, and this affects their stability conditions. The corresponding equation of motion and stability conditions are

$$
\left[t_{0}, t_{1}\right):\left\{\begin{array}{c}
\mu \text { evolution equation : } \dot{\mu}=-G^{\prime}(\mu)-(\alpha-\epsilon) H(\mu, \mu)-4 \alpha H(\mu, 0), \\
v \text { stability condition : }-G^{\prime}(0)-2 \alpha H(0, \mu)-3 \alpha H(0,0) \leqslant 0, \\
\sigma \text { stability condition : }-G^{\prime}(0)-\alpha H(0, \mu)-4 \alpha H(0,0) \leqslant 0, \\
\tau, \zeta \text { stability conditions : }-G^{\prime}(0)-5 \alpha H(0,0) \leqslant 0 .
\end{array}\right.
$$

Indeed, since $\left|F^{\prime}\right|$ is much smaller than $\left|G^{\prime}\right|$, and $\partial d / \partial \lambda$ is bounded, then $\dot{\mu}>0$ for $t \geqslant t_{0}$, which implies that $\mu(t)$ is increasing. Hence, since $F^{\prime}$ is positive and decreasing to zero, the term $-2 \alpha H(0, \mu(t)$ ) in the stability condition for $v$ (pentamers 6 and 10 ) is increasing with time, so that there exists a time $t_{1}>t_{0}$ such that the stability condition for pentamers 6 and 10 is not satisfied anymore [i.e., $-G^{\prime}(0)-2 \alpha H\left(0, \mu\left(t_{1}\right)\right)-3 \alpha H(0,0)=0$ ], while it is still satisfied for the remaining pentamers $(3,5,7,8,9$, and 11). This is true if $\alpha \leqslant \frac{5}{3} \alpha_{c}$, which is satisfied since $\alpha \leqslant \frac{5}{4} \alpha_{c}$ by (10).

Therefore, at $t=t_{1}$ pentamers 6 and 10 detach and expand for $t>t_{1}$, while none of the others is mobile yet. Again, the stability conditions for the adjacent pentamers are modified by the fact that their bonds with pentamers $1,2,6$, and 10 are weakened:

$$
\left[t_{1}, t_{2}\right):\left\{\begin{array}{l}
\mu \text { evolution equation }: \dot{\mu}=-G^{\prime}(\mu)-(\alpha-\epsilon) H(\mu, \mu)-2 \alpha H(\mu, v)-2 \alpha H(\mu, 0), \\
v \text { evolution equation }: \dot{v}=-G^{\prime}(\nu)-2 \alpha H(\nu, \mu)-3 \alpha H(v, 0), \\
\sigma \text { stability condition : }-G^{\prime}(0)-\alpha H(0, \mu)-\alpha H(0, v)-3 \alpha H(0,0) \leqslant 0 \\
\tau \text { stability condition : }-G^{\prime}(0)-\alpha H(0, v)-4 \alpha H(0,0) \leqslant 0 \\
\zeta \text { stability condition : }-G^{\prime}(0)-5 \alpha H(0,0) \leqslant 0 .
\end{array}\right.
$$

Hence, an argument analogous to the above shows that there exists a time $t_{2}$ at which the stability condition for pentamers $3,5,9$, and 11 stops being satisfied, while it is still satisfied for pentamers $4,7,8$, and 12 . For $t>t_{2}$, pentamers $1,2,3,5,6,9$, 
10, and 11 expand after detaching:

$$
\left[t_{2}, t_{3}\right):\left\{\begin{array}{l}
\mu \text { evolution equation }: \dot{\mu}=-G^{\prime}(\mu)-(\alpha-\epsilon) H(\mu, \mu)-2 \alpha H(\mu, \nu)-2 \alpha H(\mu, \sigma), \\
v \text { evolution equation : } \dot{v}=-G^{\prime}(v)-2 \alpha H(\nu, \mu)-2 \alpha H(\nu, \sigma)-\alpha H(\nu, 0), \\
\sigma \text { evolution equation : } \dot{\sigma}=-G^{\prime}(\sigma)-\alpha H(\sigma, \sigma)-\alpha H(\sigma, \mu)-\alpha H(\sigma, v)-2 \alpha H(\sigma, 0), \\
\tau \text { stability condition : }-\alpha H(0, v)-2 \alpha H(0, \sigma)-2 \alpha H(0,0) \leqslant 0, \\
\zeta \text { stability condition: }-G^{\prime}(0)-2 \alpha H(0, \sigma)-3 \alpha H(0,0) \leqslant 0 .
\end{array}\right.
$$

Again, there exists a time $t_{3}$ such that the stability condition for pentamers 4 and 12 is not satisfied, while it is still satisfied for pentamers 7 and 8 . For $t>t_{3}$, all pentamers except 7 and 8 expand after detaching, and there exists a time $t_{4}$ such that the stability condition for pentamers 7 and 8 is not satisfied:

$$
\left[t_{3}, t_{4}\right):\left\{\begin{array}{l}
\mu \text { evolution equation : } \dot{\mu}=-G^{\prime}(\mu)-(\alpha-\epsilon) H(\mu, \mu)-2 \alpha H(\mu, \nu)-2 \alpha H(\mu, \sigma), \\
\nu \text { evolution equation }: \dot{v}=-G^{\prime}(\nu)-2 \alpha H(\nu, \mu)-2 \alpha H(\nu, \sigma)-\alpha H(\nu, \tau), \\
\sigma \text { evolution equation }: \dot{\sigma}=-G^{\prime}(\sigma)-\alpha H(\sigma, \sigma)-\alpha H(\sigma, \mu)-\alpha H(\sigma, v)-\alpha H(\sigma, \tau)-\alpha H(\sigma, 0), \\
\tau \text { evolution equation : } \dot{\tau}=-G^{\prime}(\tau)-H_{\alpha}(\tau, \nu)-2 H_{\alpha}(\tau, \sigma)-2 H_{\alpha}(\tau, 0), \\
\zeta \text { stability condition : }-G^{\prime}(0)-2 \alpha H(0, \sigma)-2 \alpha H(0, \tau)-\alpha H(0,0) \leqslant 0 .
\end{array}\right.
$$

Finally, for $t_{4}<t \leqslant T$, all pentamers are detached and expanding:

$$
\left[t_{4}, T\right):\left\{\begin{array}{l}
\mu \text { evolution equation }: \dot{\mu}=-G^{\prime}(\mu)-(\alpha-\epsilon) H(\mu, \mu)-2 \alpha H(\mu, \nu)-2 \alpha H(\mu, \sigma), \\
v \text { evolution equation : } \dot{v}=-G^{\prime}(\nu)-2 \alpha H(\nu, \mu)-2 \alpha H(\nu, \sigma)-\alpha H(\nu, \tau), \\
\sigma \text { evolution equation }: \dot{\sigma}=-G^{\prime}(\sigma)-\alpha H(\sigma, \sigma)-\alpha H(\sigma, \mu)-\alpha H(\sigma, \nu)-\alpha H(\sigma, \tau)-\alpha H(\sigma, \zeta), \\
\tau \text { evolution equation : } \dot{\tau}=-G^{\prime}(\tau)-H_{\alpha}(\tau, \nu)-2 H_{\alpha}(\tau, \sigma)-2 H_{\alpha}(\tau, \zeta), \\
\zeta \text { evolution equation }: \dot{\zeta}=-G^{\prime}(\zeta)-2 H_{\alpha}(\zeta, \sigma)-2 H_{\alpha}(\zeta, \tau)-H_{\alpha}(\zeta, \zeta) .
\end{array}\right.
$$

The resulting dynamics is therefore a cascade of expansion events across the capsid surface, as illustrated in Figs. 4 and 5.

(a)
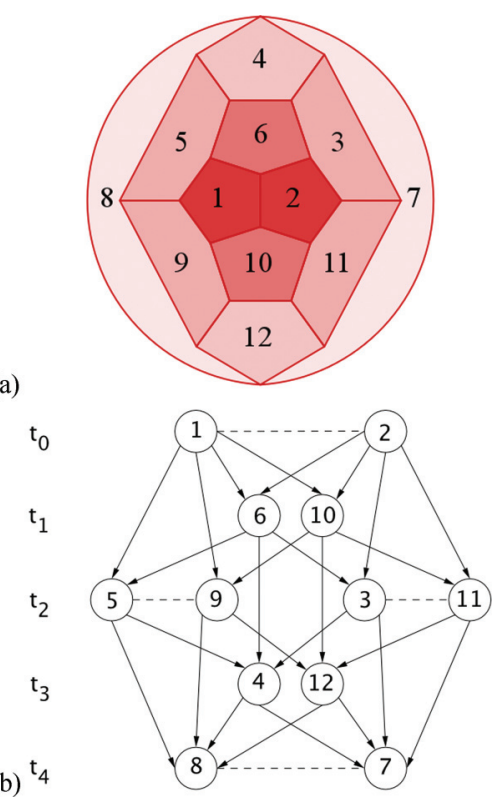

FIG. 4. (Color online) The wavelike cascade of local opening events. Panel (a) illustrates the labeling of pentamers in the capsid, seen in a planar representation along the twofold axis that corresponds to the weaker bond strength. Panel (b) illustrates the time course of the cascade, indicating the pentamers moving at any given time, together with links to the pentamers whose stability properties are affected by this motion.
Note that we did not specify the functions $F$ and $G$ explicitly and that this result hence holds in general. However, we have worked out an example explicitly in the Supplemental Material [28] and have created a movie that illustrates the expansion behavior.

\section{THE END POINT OF THE EXPANSION}

Even if the expansion intermediates have reduced symmetry, as in the case discussed above, the end state will again be icosahedral. In fact, assume that the interaction energy between the pentamers is short-range and can hence be neglected for $t>T$. In this regime, the dynamics of the system is

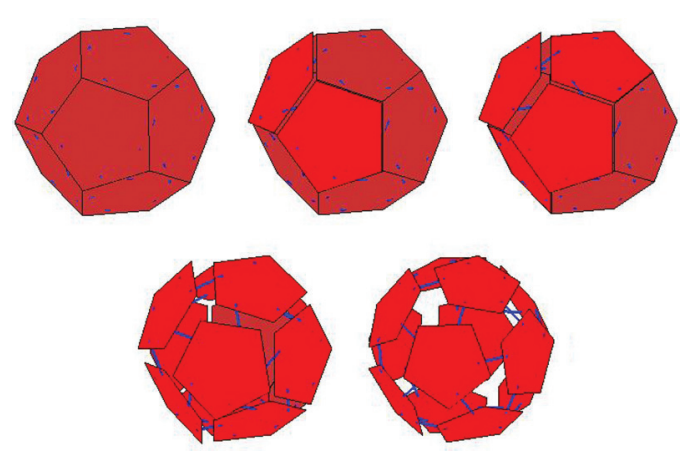

FIG. 5. (Color online) Five snapshots of the expansion event of a viral capsid. 

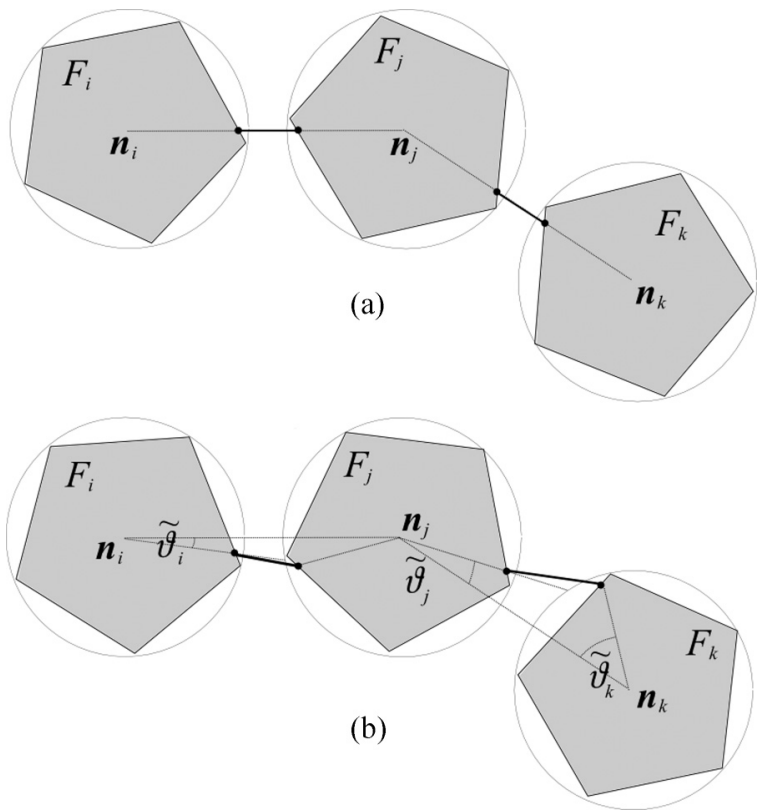

FIG. 6. A planar representation of three pentamers. Panel (a) is the symmetric equilibrium configuration: the anchor points (black dots) are aligned with the pentamer axes. Panel (b) is a generic configuration, with $\tilde{\vartheta}$ measured from the reference configuration in (a).

described by

$$
\begin{aligned}
& \dot{\lambda}_{i}=-G^{\prime}\left(\lambda_{i}\right)-n C \sum_{j \in N(i)} S\left[\left(\ell_{i j}^{2}-\ell_{0}^{2}\right)^{n-1}\right] \frac{\partial \ell_{i j}^{2}}{\partial \lambda_{i}}, \\
& \dot{\vartheta}_{i}=-n C \sum_{j \in N(i)} S\left[\left(\ell_{i j}^{2}-\ell_{0}^{2}\right)^{n-1}\right] \frac{\partial \ell_{i j}^{2}}{\partial \vartheta_{i}},
\end{aligned}
$$

where $S(x)=x$ if $x \geqslant 0$, and $S(x)=0$ otherwise.

We prove in an Appendix that there exists a unique symmetric equilibrium $\left(\lambda_{i}^{*}, \vartheta_{i}^{*}\right)$ of the dynamical system (13) of the form

$$
\lambda_{i}^{*}=\lambda^{*}, \quad \vartheta_{i}^{*}=\vartheta^{*}, \quad i=1, \ldots, 12,
$$

where $\vartheta^{*}$ is the rotation angle of the pentamers which corresponds to having all anchor points aligned with the pentamer axes $\boldsymbol{n}_{i}$ and $\boldsymbol{n}_{j}$ [cf. Fig. 6]. Numerical simulations show that this equilibrium is attractive.

\section{DISCUSSION}

Intermediates of capsid transitions are transient and hence highly difficult to capture experimentally. Theoretical input is therefore required to better understand transition events. Since the start and end state of a transition are often icosahedral, simulations usually assume icosahedral symmetry also during the transition. However, earlier work on the virus CCMV ([29-31], [15]) has shown that this is not necessarily the case, which has prompted the present analysis of a coarse-grained model system. Indeed, the analysis developed here shows that in the presence of asymmetric components or local fluctuations of the environment affecting the strengths of the interpentamer bonds, capsid expansion follows a lower symmetry path, with the symmetry of the path implied by the distribution of the weaker bonds.

We have shown here that in this situation the expansion is not, as often assumed in the literature, icosahedral, but follows a cascade of local opening events, and we explicitly determine the nature of this cascade for our model system. We moreover have shown that, irrespective of the number of weaker bonds and the explicit path taken, the end result of the expansion is again icosahedral. It is hence not surprising that imaging results regarding the start and end states of a capsid transition display icosahedral symmetry. However, the present paper shows that the inference that all intermediate states must also have this high degree of symmetry is likely to be incorrect, as suggested by [32].

More than this, we show that it is enough, and indeed energetically favorable, that a single bond is weakened below a certain threshold in order to trigger the transition and eventually drive the whole capsid to its expanded state. Hence, when the chemical environment of the capsid changes, either the presence of asymmetric components, or random local fluctuations of the bond strength, are enough to reach the destabilization threshold.

Our considerations have been based on a model system motivated by viruses such as the ERAV, in which the capsid proteins form pentagonal clusters of 20 proteins each, that play the role of disassembly units and behave as rigid domains when the capsid expands to release the genomic material $[19,26]$. Predictions regarding the expanded form are in good agreement with recent experimental results for this virus [26] and our results provide insights into the mechanisms underlying the expansion event. Viruses with capsids made by 12 pentamers and $10(T-1)$ hexamers could be treated analogously to our procedure, with state variables $\lambda_{i}$ pointing along midpoints of all capsomeres (pentamers and hexamers), each endowed in addition with a rotational degree of freedom. In principle, hexamers do undergo changes, e.g., become flatter in the expanded state as in the case of CCMV, but as a first-order effect, their radial expansion and rotation are the dominant contributions to the dynamics, and our procedure therefore could provide insights also in the expansions of these capsids.

For capsids with asymmetric components, our results demonstrate how these components can impact on the way in which the capsid becomes unstable as a prerequisite for releasing its cargo. We have discussed explicitly the case of an energy function in which a single bond has a lower bond strength, with all others being equal. This has resulted in a wavelike expansion around an axis pointing through the midpoint of the bond and the center of the capsid. Similarly, for a larger number of bonds becoming unstable simultaneously, one would expect a wavelike expansion along an axis about which this configuration is invariant. For example, if five bonds are simultaneously weakened around a fivefold axis, the wave propagates along this axis and the expansion has fivefold symmetry.

Many viruses have a portal vertex through which the viral genome is packaged. One of the open questions in structural virology is why there is canonically a mismatch in the symmetry of the portal and the pentameric capsid vertex on which it is mounted. Our analysis suggests that such a mismatch created by a packaging motor in the capsid lattice could serve as a nucleus for an expansion event of the type 
described in this paper, and expansion might hence be one of the functional consequences of the portal.

This paper hence helps to understand the mechanism of capsid transition and urges caution in the interpretation of imaging results beyond the start and end states of the expansion event. It provides insights into the nature of likely transition paths that could be used in simulation studies in combination with additional information on the exact energetic contributions. For example, explicit paths such that the one in Fig. 5 (see also the Supplemental Material [28]), could serve as input for simulations based on realistic potentials for viruses of interest. Transition paths are notoriously difficult to simulate without further input regarding the nature of the path as provided by this analysis here, and this paper could therefore open up opportunities for the simulation of transitions in viral capsids. These insights can potentially be exploited in the design of antiviral strategies that act by blocking the transition mechanism.

\section{ACKNOWLEDGMENTS}

R.T. and G.I. thank the Leverhulme Trust for financial support via a Research Leadership Award. P.C. and G.I. acknowledge the Italian PRIN 2009 project "Mathematics and Mechanics of Biological Systems and Soft Tissues."

\section{APPENDIX A: THE FINAL STATE OF THE EXPANSION}

We prove here that there exists a unique symmetric equilibrium of the dynamical system (13). Consider first the equilibrium condition for $\vartheta_{i}$ :

$$
-\sum_{j \in N(i)} S\left[\left(\ell_{i j}^{2}-\ell_{0}^{2}\right)^{n-1}\right] \frac{\partial \ell_{i j}^{2}}{\partial \vartheta_{i}}=0, \quad i=1, \ldots, 12 ;
$$

this can be satisfied only if each summand vanishes, i.e., if

$$
S\left[\left(\ell_{i j}^{2}-\ell_{0}^{2}\right)^{n-1}\right] \frac{\partial \ell_{i j}^{2}}{\partial \vartheta_{i}}=0, \quad i, j=1, \ldots, 12 .
$$

To see this, let $f_{i j}=-S\left[\left(\ell_{i j}^{2}-\ell_{0}^{2}\right)^{n-1}\right] \frac{\partial \ell_{i j}^{2}}{\partial \vartheta_{i}}$, so that the equilibrium conditions take the form $\sum_{j \in N(i)} f_{i j}=0$, and assume that, by contradiction, for some $i$, there exists $j$ such that, say, $f_{i j}<0$. Then, since

$$
\frac{\partial \ell_{i j}^{2}}{\partial \vartheta_{i}}=\frac{\partial\left|\boldsymbol{Q}_{i j}-\boldsymbol{Q}_{j i}\right|^{2}}{\partial \vartheta_{i}}=-2 \boldsymbol{n}_{i} \cdot \boldsymbol{Q}_{i j} \times \boldsymbol{Q}_{j i},
$$

it follows that $\boldsymbol{n}_{i} \cdot \boldsymbol{Q}_{i j} \times \boldsymbol{Q}_{j i}<0$, i.e., the axis of pentamer $\boldsymbol{i}$ lies on one specific side of the plane generated by the anchor points and the center of the dodecahedron [Fig. 6(b)]. By consequence, the adjacent pentamer $j$ must be rotated by an angle $\left|\tilde{\vartheta}_{j}\right|>\left|\tilde{\vartheta}_{i}\right|$, where $\tilde{\vartheta}_{i}=\vartheta_{i}-\vartheta^{*}$, with $\vartheta^{*}$ the angle corresponding to the symmetric equilibrium of Fig. 6(a), and defined below.

However, since $f_{j i}<0$ [cf. Fig. 6(b) and (A1)], there exists $k$ such that $f_{j k}>0$, and an argument similar to that above shows that the pentamer $k$ must be rotated by an angle $\left|\tilde{\vartheta}_{k}\right|>$ $\left|\tilde{\vartheta}_{j}\right|$. Iterating this argument we arrive at a contradiction since the index set is finite.

Having established that all $f_{i j}=0$, we exclude configurations such that $\ell_{i j}^{2} \leqslant \ell_{0}^{2}$ for all $i, j$, since these, when inserted in (13), would imply that $G^{\prime}\left(\lambda_{i}^{*}\right)=0$, which is excluded by our hypotheses (we want the RNA-capsid interaction force to be still active in the open form of the capsid). Hence, the equilibrium equation for $\vartheta_{i}$ reduces to

$$
\frac{\partial \ell_{i j}^{2}}{\partial \vartheta_{i}}=0 \text { for all } i, j \in 1, \ldots, 12
$$

i.e.,

$$
\boldsymbol{n}_{i} \cdot \boldsymbol{Q}_{i j} \times \boldsymbol{Q}_{j i}=\boldsymbol{n}_{j} \cdot \boldsymbol{Q}_{i j} \times \boldsymbol{Q}_{j i}=0, \quad \text { for every } i, j,
$$

which means that $\boldsymbol{Q}_{i j}$ and $\boldsymbol{Q}_{j i}$ belong to the plane through $\boldsymbol{n}_{i}$ and $\boldsymbol{n}_{j}$, so that

$$
\boldsymbol{n}_{i} \times \boldsymbol{n}_{j} \cdot \boldsymbol{Q}_{i j}=\boldsymbol{n}_{i} \times \boldsymbol{n}_{j} \cdot \boldsymbol{Q}_{j i}=0, \quad \text { for every } i, j ;
$$

i.e.,

$$
\boldsymbol{n}_{i} \times \boldsymbol{n}_{j} \cdot R_{i}\left(\vartheta_{i}\right) \boldsymbol{P}_{i j}=\boldsymbol{n}_{i} \times \boldsymbol{n}_{j} \cdot R_{j}\left(\vartheta_{i}\right) \boldsymbol{P}_{j i}=0,
$$

for every $i, j$. This expression is independent of $\lambda_{i}, \lambda_{j}$, and its unique solution $\vartheta_{i}=\vartheta_{j}=\vartheta^{*}$ corresponds to having the anchor points aligned with the plane through $\boldsymbol{n}_{1}$ and $\boldsymbol{n}_{2}$, the pentamers axes. Therefore, the solution of the second equilibrium equation is $\vartheta_{i}=\vartheta^{*}$ for all $i$.

We now prove that all the solutions of the first equilibrium equation for $\vartheta_{i}=\vartheta^{*}$ are of the form $\lambda_{i}=\lambda^{*}$ for all $i$, which, in turn, implies that the final state of the expansion is icosahedral irrespective of the nature of the transition path. Consider, in fact, the system

$$
-G^{\prime}\left(\lambda_{i}\right)=n C \sum_{j \in N(i)}\left(\ell_{i j}^{2}-\ell_{0}^{2}\right)^{n-1} \frac{\partial \ell_{i j}^{2}}{\partial \lambda_{i}}, \quad i=1, \ldots, 12,
$$

for $n=2$ and $\vartheta_{i}=\vartheta_{j}=\vartheta^{*}$, where

$$
\frac{\partial \ell_{i j}^{2}}{\partial \lambda_{i}}=\frac{\partial\left|\boldsymbol{Q}_{i j}-\boldsymbol{Q}_{j i}\right|^{2}}{\partial \lambda_{i}}=2\left(\boldsymbol{Q}_{i j}-\boldsymbol{Q}_{j i}\right) \cdot \boldsymbol{n}_{i} .
$$

Let $\lambda_{0}$ and $\psi$ be defined as in Fig. 7, and $\varrho_{i}=\lambda_{i}-\lambda_{0}$ : then

$$
\begin{aligned}
& \ell^{2}\left(\lambda_{i}, \lambda_{j}, \vartheta^{*}, \vartheta^{*}\right) \\
& \quad=\left(\lambda_{i}-\lambda_{j}\right)^{2}+2\left(\lambda_{i}-\lambda_{0}\right)\left(\lambda_{j}-\lambda_{0}\right)(1-\cos \psi) \\
& \quad=\left(\varrho_{i}-\varrho_{j}\right)^{2}+2 \varrho_{i} \varrho_{j}(1-\cos \psi),
\end{aligned}
$$

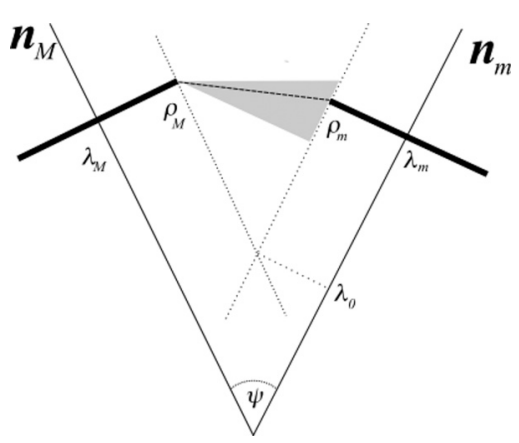

FIG. 7. Cross section of the dodecahedral model of the capsid showing the maximally and minimally expanded pentamers. Thick lines are side views of the pentamers. The shaded area corresponds to $\varrho_{M} \cos \psi<\varrho_{m}<\varrho_{M}$. 
and, when $\vartheta_{i}=\vartheta_{j}=\vartheta^{*}$,

$$
\begin{aligned}
\left(\boldsymbol{Q}_{i j}-\boldsymbol{Q}_{j i}\right) \cdot \boldsymbol{n}_{i} & =\left(\lambda_{i}-\lambda_{0}\right)-\left(\lambda_{j}-\lambda_{0}\right) \cos \psi \\
& =\varrho_{i}-\varrho_{j} \cos \psi .
\end{aligned}
$$

Assume now that there exist $\varrho_{m}<\varrho_{M}$ such that $\varrho_{m} \leqslant$ $\varrho_{k} \leqslant \varrho_{M}$ for every $k=1, \ldots, 12$. Since $-G^{\prime}$ is monotone decreasing,

$$
-G^{\prime}\left(\lambda_{m}\right)>-G^{\prime}\left(\lambda_{M}\right)
$$

which implies

$$
\begin{aligned}
& \sum_{j \in N(m)}\left(\ell_{m j}^{2}-\ell_{0}^{2}\right)\left(\varrho_{m}-\varrho_{j} \cos \psi\right) \\
& >\sum_{k \in N(M)}\left(\ell_{M k}^{2}-\ell_{0}^{2}\right)\left(\varrho_{M}-\varrho_{k} \cos \psi\right) ;
\end{aligned}
$$

i.e.,

$$
\begin{aligned}
& \sum_{k \in N(M)}\left(\ell_{M k}^{2}-\ell_{0}^{2}\right) \varrho_{M}-\sum_{j \in N(m)}\left(\ell_{m j}^{2}-\ell_{0}^{2}\right) \varrho_{m} \\
& \quad<\cos \psi\left(\sum_{k \in N(M)}\left(\ell_{M k}^{2}-\ell_{0}^{2}\right) \varrho_{k}-\sum_{j \in N(m)}\left(\ell_{M j}^{2}-\ell_{0}^{2}\right) \varrho_{j}\right) \\
& \quad \leqslant \cos \psi\left(\sum_{k \in N(M)}\left(\ell_{M k}^{2}-\ell_{0}^{2}\right) \varrho_{M}-\sum_{j \in N(m)}\left(\ell_{M j}^{2}-\ell_{0}^{2}\right) \varrho_{m}\right) .
\end{aligned}
$$

Assume now that $\varrho_{m}>\varrho_{M} \cos \psi$ : then $\sum_{k \in N(M)}\left(\ell_{M k}^{2}-\right.$ $\left.\ell_{0}^{2}\right) \varrho_{M} \geqslant \sum_{j \in N(m)}\left(\ell_{m j}^{2}-\ell_{0}^{2}\right) \varrho_{m}$, which implies that the left- hand side of (A3) is non-negative. In fact,

$$
\min _{j \in N(M)} \ell_{M j}^{2}=\ell_{M m}^{2}=\max _{j \in N(m)} \ell_{m j}^{2} .
$$

Hence, since $\cos \psi<1$, (A3) is impossible and $\lambda_{m}=\lambda_{M}$.

\section{APPENDIX B: A SPECIFIC EXAMPLE}

We have worked out a concrete example explicitly in order to create a movie of a sample transition path. Using the following functions for $F$ and $G$ in the expression of the total energy (see also Fig. 3):

$$
\begin{aligned}
& F(x)= \begin{cases}-\frac{a}{2 b^{2}}(x-b)^{2}, & x \in[0, b], \\
0, & \text { otherwise, }\end{cases} \\
& G(x)= \begin{cases}\frac{g}{2 f^{2}}(x-f)^{2}, & x \in[0, f], \\
0, & \text { otherwise, }\end{cases}
\end{aligned}
$$

with $b \ll f$, and we have chosen the following form for the intercapsomere distance

$$
d\left(\lambda, \lambda^{\prime}, \vartheta, \vartheta^{\prime}\right)=\lambda+\lambda^{\prime}+q\left[\vartheta^{2}+\left(\vartheta^{\prime}\right)^{2}-p \vartheta \vartheta^{\prime}\right]
$$

A few snapshots of this dynamics are shown in Fig. 5.

Note that the transition intermediates have twofold symmetry and that the expansion spreads like a cascade of local events over the capsid surface, starting at the weaker bond on one of the edges between pentamers and finishing at the opposite edge.
[1] F. H. Crick and J. D. Watson, Nature (London) 177, 473 (1956).

[2] D. L. D. Caspar and A. Klug, Cold Spring Harb. Symp. Quant. Biol. 27, 1 (1962).

[3] I. Robinson and S. Harrison, Nature (London) 297, 563 (1982).

[4] M. B. Sherman, H. R. Guenther, F. Tama, T. L. Sit, C. L. Brooks, A. M. Mikhailov, E. V. Orlova, T. S. Baker, and S. A. Lommel, J. Virol. 80, 10395 (2006).

[5] J. A. Speir, S. Munshi, G. Wang, T. S. Baker, and J. E. Johnson, Structure 3, 63 (1995).

[6] J. E. Johnson, Curr. Opin. Struct. Biol. 20(2), 210 (2010).

[7] R. W. Hendrix and J. E. Johnson, Adv. Exp. Med. Biol. 726, 351 (2012).

[8] M. Kim, R. Jernigan, and G. Chirikjian, J. Struct. Biol. 143, 107 (2003).

[9] F. Tama and C. L. Brooks III, J. Mol. Biol. 345, 299 (2005).

[10] D. Veesler, J. Quispe, N. Grigorieff, C. Potter, B. Carragher, and J. Johnson, Structure 20, 1384 (2012).

[11] W. Roos, I. Gertsman, E. May, C. L. Brooks, III, J. Johnson, and G. Wuite, Proc. Natl. Acad. Sci. U.S.A. 109, 2342 (2012).

[12] E. May, J. Feng, and C. L. Brooks, III, Biophys. J. 102, 606 (2012).

[13] E. May and C. L. Brooks, III, J. Phys. Chem. B 116, 8604 (2012).

[14] F. Tama and C. L. Brooks, III, J. Mol. Biol 318, 733 (2002).
[15] G. Indelicato, P. Cermelli, D. G. Salthouse, S. Racca, G. Zanzotto, and R. Twarock, J. Math. Biol. 64, 745 (2012).

[16] J. Conway, W. Wikoff, N. Cheng, R. Duda, R. Hendrix, J. Johnson, and A. Steven, Science 292, 744 (2001).

[17] A. Zlotnick, J. Mol. Biol. 241, 59 (1994).

[18] E. C. Dykeman, P. G. Stockley, and R. Twarock, Phys. Rev. E 87, 022717 (2013).

[19] T. Tuthill, K. Harlos, T. Walter, N. Knowles, E. Groppelli, D. Rowlands, D. Stuart, and E. Fry, PLoS Pathog. 5, e1000620 (2009).

[20] T. Aleksiev, R. Potestio, F. Pontiggia, S. Cozzini, and C. Micheletti, Bioinformatics 25, 2743 (2009).

[21] http://viperdb.scripps.edu/.

[22] T. Guérin and R. F. Bruinsma, Phys. Rev. E 76, 061911 (2007).

[23] W. H. Roos, R. Bruinsma, and G. J. L. Wuite, Nat. Phys. 6, 733 (2010).

[24] A. Aggarwal, J. Rudnick, R. F. Bruinsma, and W. S. Klug, Phys. Rev. Lett. 109, 148102 (2012).

[25] M. Kim, R. Jernigan, and G. Chirikjian, Biophys. J. 89, 43 (2005).

[26] S. E. Bakker, E. Groppelli, A. R. Pearson, P. G. Stockley, D. J. Rowlands, and N. A. Ranson, J. Virol. (to be published).

[27] K. C. Dent, R. Thompson, A. Barker, J. Barr, J. Hiscox, P. Stockley, and N. Ranson, Structure 21, 1225 (2013). 
[28] See Supplemental Material at http://link.aps.org/supplemental/ 10.1103/PhysRevE.88.032710 for a movie that illustrates the expansion behavior.

[29] T. Keef, J. Wardman, N. A. Ranson, P. G. Stockley, and R. Twarock, Acta Cryst. A 69, 140 (2013).
[30] T. Keef and R. Twarock, in Emerging Topics in Physical Virology (Imperial College Press, London, 2010), pp. 59-83.

[31] T. Keef and R. Twarock, J. Math. Biol. 59, 287 (2009).

[32] A. Steven (private communication). 